\title{
Research and development for accuracy improvement of neutron nuclear data on minor actinides
}

\author{
Hideo Harada ${ }^{1}$ a, Osamu Iwamoto ${ }^{1}$, Nobuyuki Iwamoto ${ }^{1}$, Atsushi Kimura ${ }^{1}$, Kazushi Terada ${ }^{1}$, Taro Nakao ${ }^{1}$, \\ Shoji Nakamura ${ }^{1}$, Kazuhito Mizuyama ${ }^{1}$, Masayuki Igashira ${ }^{2}$, Tatsuya Katabuchi ${ }^{2}$, Tadafumi Sano ${ }^{3}$, Yuji Shibahara ${ }^{3}$, \\ Yoshiyuki Takahashi ${ }^{3}$, Koichi Takamiya ${ }^{3}$, Cheol Ho Pyeon ${ }^{3}$, Satoshi Fukutani ${ }^{3}$, Tosiyuki Fujii ${ }^{3}$, Jun-ichi Hori ${ }^{3}$, \\ and Hiroshi Yashima ${ }^{3}$ \\ 1 Japan Atomic Energy Agency, Nuclear Science and Engineering Center, Tokai, Naka, Ibaraki 319-1195, Japan
2 Tokyo Institute of Technology, Laboratory for Advanced Nuclear Energy, O-okayama, Meguro, Tokyo 152-8550, Japan
3 Kyoto University, Research Reactor Institute, Kumatori, Sennan, Osaka 590-0494, Japan
}

\begin{abstract}
To improve accuracy of neutron nuclear data on minor actinides, a Japanese nuclear data project entitled "Research and development for Accuracy Improvement of neutron nuclear data on Minor ACtinides (AIMAC)" has been implemented. Several independent measurement techniques were developed for improving measurement precision at J-PARC/MLF/ANNRI and KURRI/LINAC facilities. Effectiveness of combining the independent techniques has been demonstrated for identifying bias effects and improving accuracy, especially in characterization of samples used for nuclear data measurements. Capture cross sections and/or total cross sections have been measured for Am-241, Am-243, Np-237, Tc-99, Gd-155, and Gd-157. Systematic nuclear data evaluation has also been performed by taking into account the identified bias effect. Highlights of the AIMAC project are outlined.
\end{abstract}

\section{Introduction}

For the study of nuclear transmutation systems, accurate nuclear data are indispensable [1-3]. As an example, sensitivity and uncertainty analysis has been performed $[4,5]$ recently for the accelerator-driven system (ADS) proposed by the Japan Atomic Energy Agency (JAEA) using the covariance of JENDL-4.0 [6]. The impact of improving accuracy of relevant nuclear data was quantitatively shown. According to the investigation, capture cross sections of minor actinides (MAs) are among the most important data. To improve accuracy of capture cross sections of MAs, a Japanese nuclear data project entitled "Research and development for Accuracy Improvement of neutron nuclear data on Minor ACtinides (AIMAC)" has been performed since October 2013 [7]. The AIMAC project focuses not only on the fast neutron region but also thermal and resonance energy regions, since both of them are important for normalization of energy dependent data measured by a TOF method.

Recently, high quality energy dependent data on capture cross sections of MAs have been measured at J-PARC/ANNRI [8-10], LANL/DANCE [11,12], CERN/n_TOF $[13,14]$ et al. Resonance analysis of neutron total cross section has also been used to deduce a capture cross section at JRC-Geel/GELINA [15]. Since statistical uncertainty of neutron time-of-flight data has been decreased drastically owing to high-intensity pulsed neutron beam produced by a spallation reaction, determination of absolute value of energy dependent data is becoming a key issue for improving the accuracy.

a e-mail: harada.hideo@jaea.go.jp
International activity by OECD/NEA for improving the accuracy has been also going on [16].

For accurate determination of the absolute value, it is important to develop high-precision measurement techniques, identify un-recognized bias effects, and correct the effects precisely. In the AIMAC project, plural independent measurement techniques have been developed in order to make cross-checks of the measurements, and to recognize bias effects that are difficult to be identified by a sole measurement technique. Finally, all of available experimental data are re-evaluated with systematic corrections of identified bias effects.

The actions of the AIMAC project are summarized as follows:

- Precise characterization of sample

- Activation measurement method at KURRI/LINAC TOF facility

- TOF measurement method at J-PARC/ANNRI and KURRI/LINAC facilities

- Re-evaluations with systematic corrections.

Highlights of the above actions are summarized in this paper. Details of each achievement are described in each contributing papers.

\section{Highlights of AIMAC project}

\subsection{Precise characterization of sample}

Accurate determination of absolute sample amount is important for cross section measurements. In the case of the sealed ${ }^{241} \mathrm{Am}$ sample previously used at ANNRI [10], the uncertainty of the sample amount was not given. 


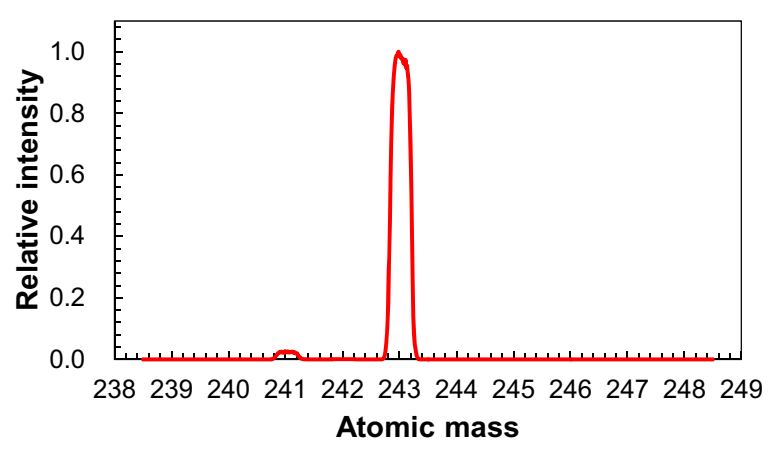

Figure 1. Mass spectrum of ${ }^{243} \mathrm{Am}$ material measured by TIMS.

Table 1. Isotopic ratio measurement of the ${ }^{243} \mathrm{Am}$ material.

\begin{tabular}{|l|c|l|}
\hline Isotopes & Declared ratio & mass spectroscopy \\
\hline${ }^{241} \mathrm{Am}$ & $2.7 \%$ & $2.29 \pm 0.03 \%$ \\
\hline${ }^{243} \mathrm{Am}$ & $97.3 \%$ & $97.71 \pm 0.03 \%$ \\
\hline
\end{tabular}

Therefore, only energy dependence of capture cross section has been determined [10]. In the AIMAC project, two sealed samples of ${ }^{241} \mathrm{Am}$ (480 and $\left.950 \mathrm{MBq}\right)$ and three sealed samples of ${ }^{243} \mathrm{Am}(60,120,240 \mathrm{MBq})$ have been prepared. Although $\gamma$-ray intensity for each sample is declared in the specification sheets, its uncertainty is as large as $10 \%$. For precise characterization, small amounts $\left(130 \mathrm{kBq}\right.$ of ${ }^{241} \mathrm{Am}$ and $10 \mathrm{kBq}$ of $\left.{ }^{243} \mathrm{Am}\right)$ of the same materials used for fabricating these sealed samples have been prepared for destructive analyses by mass spectroscopy and $\alpha$-ray spectroscopy. To determine absolute amounts of the sample, several precise nondestructive methods have also been developed, that is, micro calorimetry and $\gamma$-ray spectroscopy. Effectiveness of synthesized analyses obtained by the developed independent techniques is shown.

\subsubsection{Isotopic ratio measurement by mass spectroscopy}

The isotopic ratios of $\mathrm{Am}$ in the prepared ${ }^{241} \mathrm{Am}$ and ${ }^{243} \mathrm{Am}$ materials were measured by TIMS [17]. The isotopic purity of ${ }^{241} \mathrm{Am}$ material was verified by the TIMS: impurity level is less than 5ppm. On the other hand, isotopic impurity of ${ }^{241} \mathrm{Am}$ in the ${ }^{243} \mathrm{Am}$ material is significant. Figure 1 shows a relative intensity of Am isotopes in the ${ }^{243} \mathrm{Am}$ material. A peak corresponding to ${ }^{241} \mathrm{Am}$ is clearly shown together with a prominent ${ }^{243} \mathrm{Am}$ peak. The measured isotopic ratio is shown in Table 1. It is remarkable that extremely high precision data is obtained by this mass spectroscopy. Details of the spectroscopic techniques are described in [18].

\subsubsection{Characterization of sample materials by $\alpha$ spectroscopy}

The same ${ }^{243} \mathrm{Am}$ material has been analysed by $\alpha$ spectroscopy. Figure 2 clearly shows not only ${ }^{241} \mathrm{Am}$ and ${ }^{243} \mathrm{Am}$ but also ${ }^{242} \mathrm{Cm}$ and ${ }^{244} \mathrm{Cm}$. Since the half-lives of these $\mathrm{Cm}$ isotopes are shorter than those of Am isotopes, peaks of $\mathrm{Cm}$ isotopes are significant although $\mathrm{Cm}$ amount is much smaller than that of Am. The quantified amounts of $\mathrm{Cm}$ isotopes are indispensable for analysis of micro calorimetry [18] described in 2.1.4.

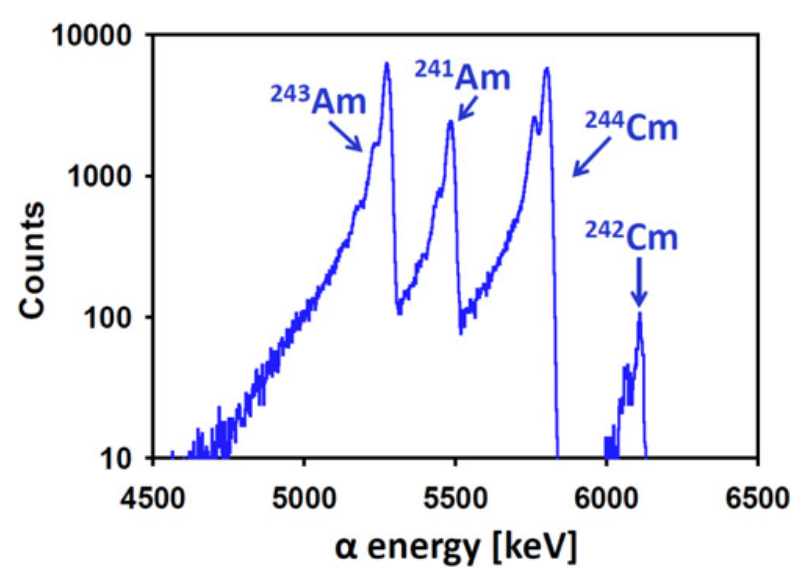

Figure 2. $\alpha$ spectrum of ${ }^{243} \mathrm{Am}$ sample material.

Table 2. Summary of quantified sample activities.

\begin{tabular}{|c|c|c|c|}
\hline Sample ID & $\begin{array}{c}\text { Declared } \\
\text { activity } \\
{[\mathrm{MBq}]}\end{array}$ & $\begin{array}{c}\gamma \text {-ray } \\
\text { spectroscopy } \\
{[\mathrm{MBq}]}\end{array}$ & $\begin{array}{c}\text { micro } \\
\text { calorimetry } \\
{[\mathrm{MBq}]}\end{array}$ \\
\hline${ }^{241} \mathrm{Am} \mathrm{\# 1}$ & 480 & $511 \pm 10$ & $510.7 \pm 0.5$ \\
\hline${ }^{241} \mathrm{Am} \mathrm{\# 2}$ & 950 & $962 \pm 19$ & $957.4 \pm 0.5$ \\
\hline${ }^{243} \mathrm{Am} \mathrm{\# 1}$ & 60 & $66.7 \pm 1.3$ & $67.3 \pm 0.3$ \\
\hline${ }^{243} \mathrm{Am} \mathrm{\# 2}$ & 120 & $155 \pm 3$ & $155.8 \pm 0.3$ \\
\hline${ }^{243} \mathrm{Am} \mathrm{\# 3}$ & 240 & $286 \pm 6$ & $281.8 \pm 0.3$ \\
\hline
\end{tabular}

\subsubsection{Absolute amount measurement by $\gamma$-ray spectroscopy}

The absolute MA amounts in the MA samples could be determined by measuring decay $\gamma$-rays escaping from the sealed samples. Gamma rays emitted from the samples were measured with a planar type High-Purity Germanium (HPGe) detector. An efficiency curve of the HPGe detector was determined with uncertainties less than about $1 \%$ by combining measured efficiencies and Monte Carlo simulation. This method was originally developed in [19]. The $\gamma$-ray emission probabilities for the major $\gamma$ rays of relevant nuclides were determined with uncertainties less than $1.2 \%$ [20].

The sample amounts deduced using the efficiency and the $\gamma$-ray emission probabilities are determined with uncertainty of about $2 \%$. The results are summarized in Table 2, together with those determined by micro calorimetry described in 2.1.4 [21].

\subsubsection{Absolute amount measurement by micro calorimetry}

A high-performance calorimeter TAM VI (Thermometric $\mathrm{AB}$, Sweden) was utilized to measure decay heat from a sealed sample. Since the Q-values and half-lives of ${ }^{241} \mathrm{Am}$ and ${ }^{243} \mathrm{Am}$ isotopes are well known with high precision, it is possible to deduce the absolute activity from the heat measurement. In the measurement, the sample was sandwiched by metallic tungsten plates to prevent decay $\gamma$-rays and X-rays escaping from the calorimeter's cell. In the case of ${ }^{243} \mathrm{Am}$ samples, contamination of $\mathrm{Cm}$ isotopes needs to be corrected as mentioned in 2.1.2. The isotopic and activity ratios deduced in 2.1.1 and 2.1.2 were used to deduce the absolute activities of ${ }^{241,243} \mathrm{Am}$ in the sealed samples. As shown in Table 2, the activities of ${ }^{241} \mathrm{Am}$ samples were determined with precision of about 
$0.05-0.1 \%$. In case of ${ }^{243} \mathrm{Am}$ samples, the achieved precision was about $0.1-0.5 \%$ because of correction uncertainty of $\mathrm{Cm}$. Contamination of $\mathrm{Pu}$ isotopes needs to be checked before obtaining final results, although its contribution is expected to be small $[18,21]$.

\subsection{Activation measurement method at KURRI TOF facility}

A neutron activation method has been developed in the neutron target room at KURRI electron linear accelerator facility. This compact facility has been used for cross section measurements using a neutron TOF technique. Neutrons are produced via a photonuclear reaction by the pulsed electron beam. The neutron producing target is set in the water tank as shown in Fig. 3. Neutrons thermalized in the tank are used for irradiating a sample set near the tank.

There are important advantages compared to measurements using research reactors as below:

- Energy dependence of neutron flux is determined experimentally using a TOF technique.

- Neutron energy spectrum is varied by adjusting the amount of boric acid in the water tank.

Activation measurements have been performed using unsealed ${ }^{237} \mathrm{~Np}$ samples. Neutron flux at the sample position was monitored using thin Au foils. It was shown that enough amount of ${ }^{238} \mathrm{~Np}$ was produced for capture cross section determination: statistical uncertainty is less than $1 \%$. Details of the activation techniques are described in [22]. Unsealed ${ }^{241} \mathrm{Am}$ and ${ }^{243} \mathrm{Am}$ samples have also been measured, and the analyses is ongoing.

\subsection{TOF measurement methods at J-PARC/ANNRI and KURRI/LINAC}

\subsubsection{Capture cross sections of ${ }^{155,157} \mathrm{Gd}$ at $J-P A R C I A N N R I$}

For absolute determination of capture cross sections, a new method was developed and applied to ${ }^{155,157} \mathrm{Gd}$ [23]. This method does not need precise values on neutron flux nor detector efficiency, but needs only a ratio of capture yields for two different thickness samples. This method is applicable in the case that the ratio of capture cross section to total cross section can be approximated by one. Figure 4 shows a part of the capture cross section of ${ }^{157} \mathrm{Gd}$ determined by this method, together with that of JENDL-4.0 [6]. The obtained data is fitted by introducing a negative resonance in resonance analyses. Details of the resonance analyses are described in [24].

\subsubsection{Capture cross sections of ${ }^{241,243} A m$ at $J-P A R C I A N N R I$}

Capture cross sections of ${ }^{241,243}$ Am have been measured using the Ge spectrometer at J-PARC/ANNRI. The samples with different thickness have been used for both ${ }^{241} \mathrm{Am}$ and ${ }^{243} \mathrm{Am}$ measurements. All of the samples are precisely characterized by the methods as described in 2.1 .

The neutron pulse at J-PARC/MLF has been generated in a double bunch mode: the time interval between two bunches is about $600 \mathrm{~ns}$ [25]. This made data analyses of ANNRI very complicated. During the measurement period

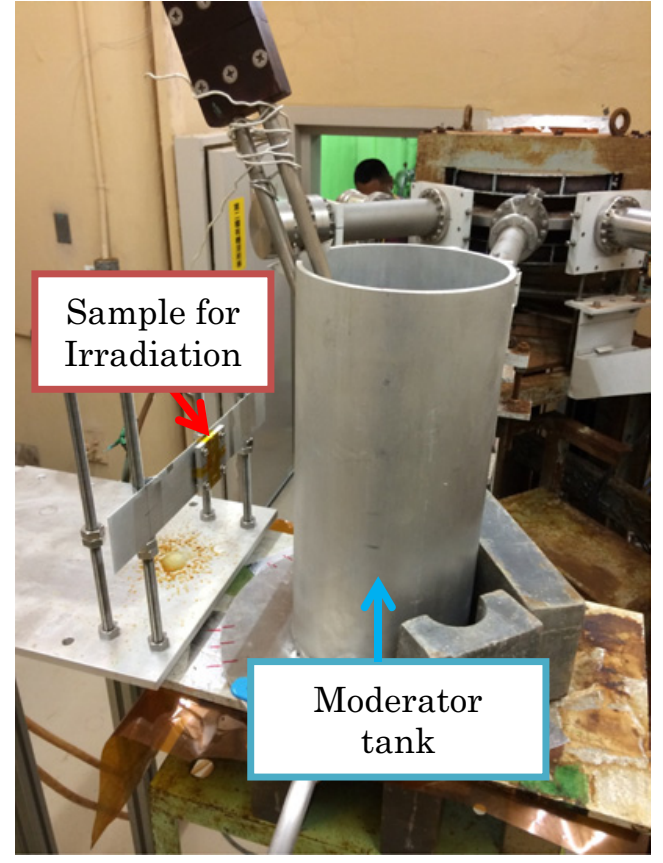

Figure 3. A photograph of activation measurement setup installed in the neutron target room at KURRI-LINAC facility.

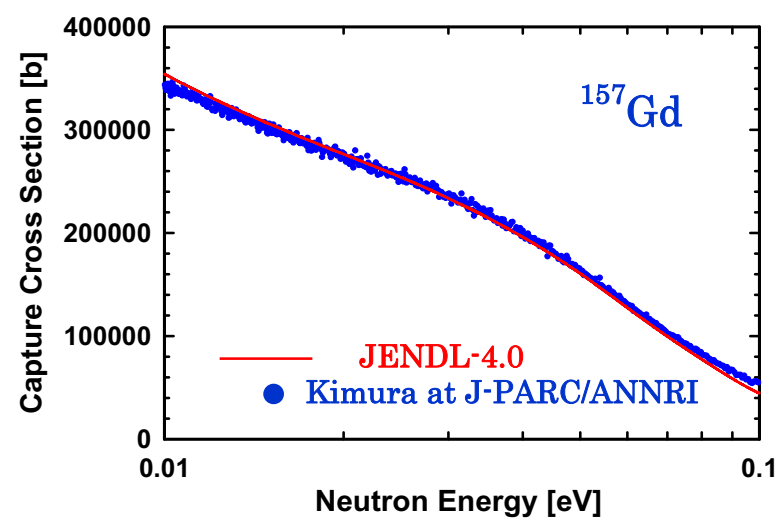

Figure 4. A low energy part of capture cross section of ${ }^{157} \mathrm{Gd}$ measured at J-PARC/ANNRI and JENDL-4.0.

of the AIMAC project, J-PARC has been operated by a single-mode. Figure 5 shows capture yields as a function of neutron energy obtained for a ${ }^{243} \mathrm{Am}$ measurement as an example. As a result, the double-bunch time structure is not observed in the figure, and resonance peaks are clearly shown even at around $100 \mathrm{eV}$.

To deduce capture cross sections from the data, neutron self-shielding and multiple-scattering effects in a sample need to be corrected. The combinational use of capture yields measured for different thickness samples is expected to enable a cross-check of the correction factor.

\subsubsection{Total cross section measurements of Am at J-PARCIANNRI}

A neutron detection system has been installed at $\mathrm{J}$ PARC/ANNRI in order to measure total cross sections. The neutron detection system is composed of a ${ }^{6} \mathrm{Li}$-glass scintillator, a ${ }^{7} \mathrm{Li}$-glass scintillator and a new DAQ system. The ${ }^{7} \mathrm{Li}$-glass scintillator, that is not sensitive to neutrons, is used to evaluate $\gamma$-ray backgrounds counted by the 


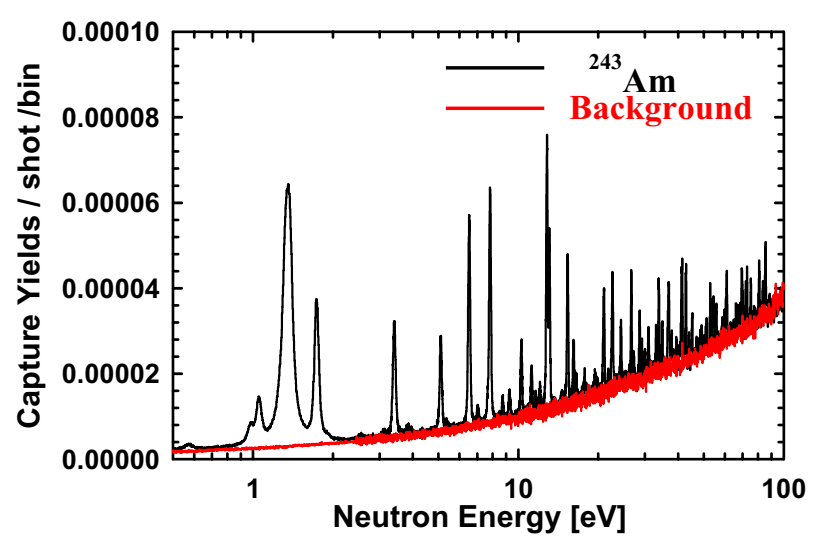

Figure 5. Capture yields for a ${ }^{243} \mathrm{Am}$ sample measured by the $\mathrm{Ge}$ spectrometer at J-PARC/ANNRI with single-bunch mode.

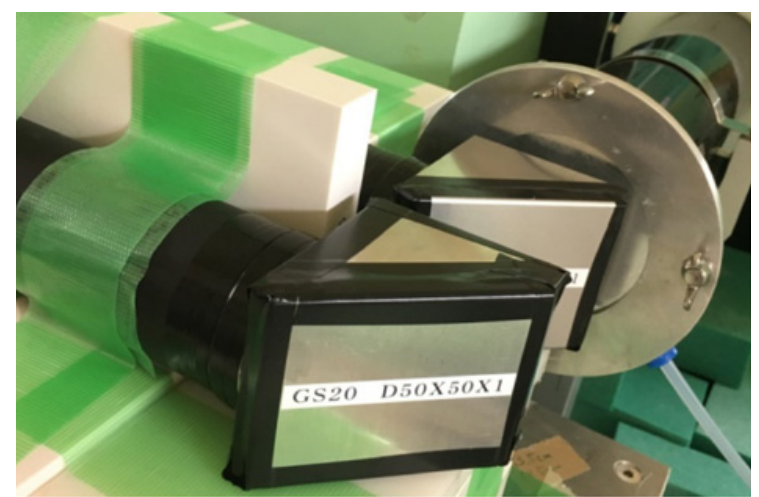

Figure 6. A photograph of neutron detectors installed in the J-PARC/ANNRI for neutron transmission measurements.

${ }^{6} \mathrm{Li}$-glass scintillator. These detectors are set at about 45degree angle to the neutron beam axis as shown in Fig. 6.

The developed DAQ system is designed to process signals from both the installed neutron detectors and the Ge spectrometer. The charge-sensitive ADC module of CAEN (model v1720, 12-bit, 250-MHz) is used for processing signals of neutron detectors. The peak-hold ADC boards of CAEN (model v1724, 14-bit, 100-MHz) is used for processing signals of the Ge spectrometer. Details on the performance of the DAQ system are described in [26].

Figure 7 shows a neutron transmission spectrum measured for a ${ }^{241} \mathrm{Am}$ sample $(957.4 \pm 0.5 \mathrm{MBq})$ using the developed neutron detection system. This is the first neutron transmission data for a MA sample at J-PARC/ANNRI. Since the ratio of capture cross section to total cross section is nearly one at low energy region, the data will be usefully used for cross-checking with the capture data on ${ }^{241} \mathrm{Am}$.

\subsubsection{Extension of energy region of capture cross section above $100 \mathrm{keV}$ at J-PARCIANNRI}

In order to extend the measurable energy region of capture cross section at ANNRI from $100 \mathrm{keV}$ to more than $300 \mathrm{keV}$, the data-taking system developed for the ANNRI$\mathrm{NaI}$ spectrometer has been upgraded. Furthermore, a neutron shield tube has been installed at the sample position to reduce neutron background [27].

The measured neutron capture cross section of ${ }^{99} \mathrm{Tc}$ is shown in Fig. 8. It is noticeable that the measurable

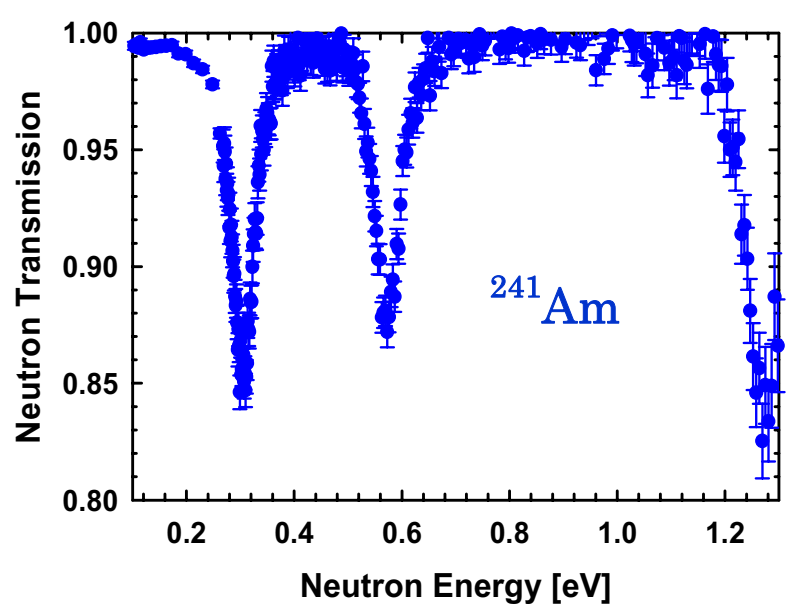

Figure 7. Neutron transmission spectrum of ${ }^{241} \mathrm{Am}$.

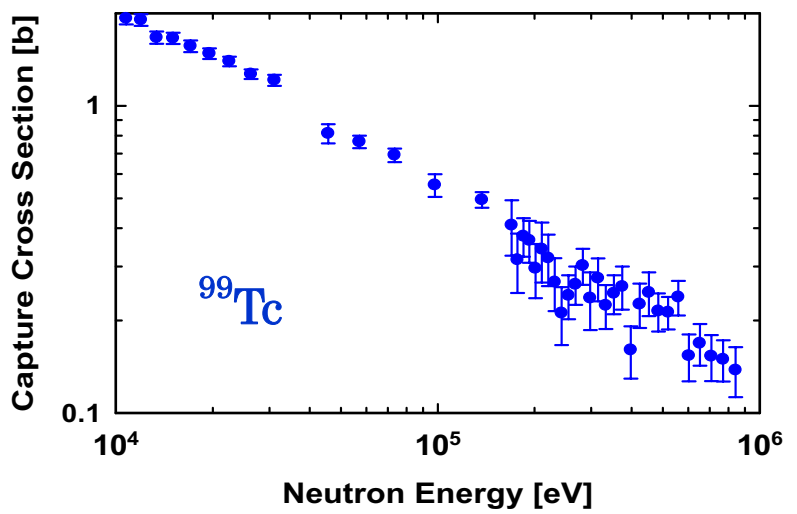

Figure 8. Neutron capture cross section of ${ }^{99} \mathrm{Tc}$ measured using the NaI spectrometer at J-PARC/ANNRI.

energy region is successfully extended from $100 \mathrm{keV}$ up to about $800 \mathrm{keV}$. Details of the measurement techniques are described in [28].

\subsubsection{TOF measurements at Kyoto/LINAC}

A $4 \pi$ BGO spectrometer has been developed and installed at the $10 \mathrm{~m}$ flight-path area in the KURRI-LINAC facility for capture cross section measurements as shown in Fig. 9. A DAQ system is also developed to process signals from the spectrometer. The high-speed digitizer of Agilent Technology (model M9210A, 10-bit) is used for processing signals of BGO detectors.

The capture cross section of ${ }^{237} \mathrm{~Np}$ has been measured using the BGO spectrometer [22]. The samples with different thickness have been used to enable cross-check of the correction factor of neutron self-shielding and multiple-scattering effect in the sealed ${ }^{237} \mathrm{~Np}$ samples. For resonance analyses, the neutron pulse resolution function of this facility has been investigated based on Monte Carlo calculations. Details of the simulation techniques are described in [29].

\subsection{Re-evaluation with systematic corrections}

\subsubsection{Evaluations of capture cross sections for the thermal neutron region}

For the measurement of thermal neutron capture cross sections, an activation method has been widely used using 


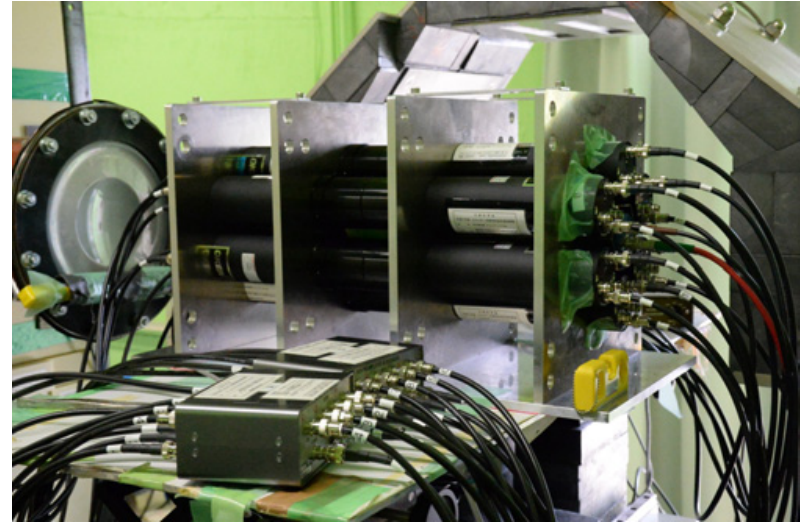

Figure 9. A photograph of $4 \pi \mathrm{BGO}$ spectrometer installed in the KURRI-LINAC facility.

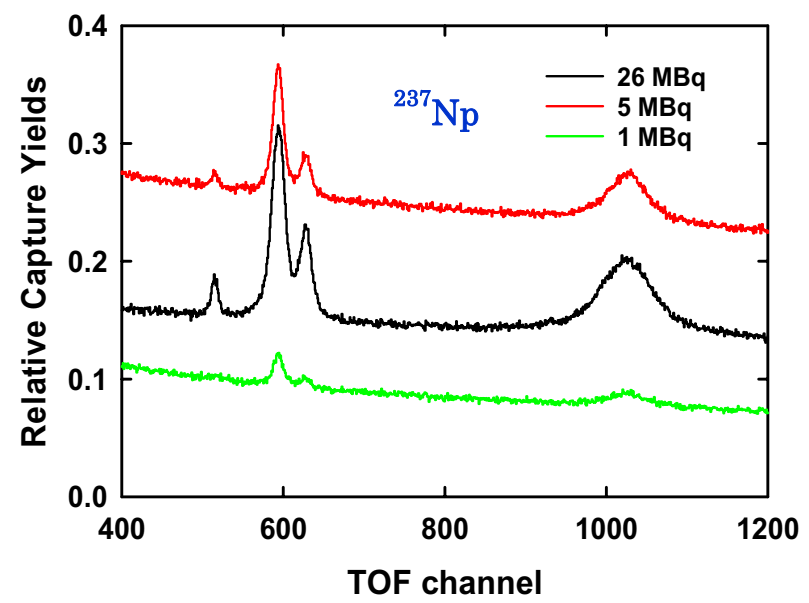

Figure 10. Capture yields for three ${ }^{237} \mathrm{~Np}$ samples measured by the BGO spectrometer at KURRI/LINAC.

a research reactor [30-32]. In order to separate thermal neutrons from epi-thermal neutrons, a Cd cut-off method has been applied. The Cd cut-off energy is typically about $0.5 \mathrm{eV}$. In the case of MAs, large resonances are located just near the cut-off energy. In order to deduce thermal capture cross sections accurately, the reaction rate near the Cd cut-off energy needs to be evaluated carefully. Some of the old measurements did not take into account this effect. Recently, systematic corrections have been performed using the identified systematic bias effect $[33,34]$. An example of the systematic correction performed in the AIMAC project on ${ }^{241} \mathrm{Am}$ is shown in Fig. 11 (data in upper graph is before correction, and that in lower after correction). The limited data are corrected where enough information is given for the correction. The other type of measurememnts are also shown in the same figure for comparison. It is remarkable that the dispersion of data is drastically reduced after this systematic correction.

\subsubsection{Evaluations of capture cross sections for fast neutron region}

In order to evaluate capture cross sections for fast neutron region, statistical model calculations have been performed. For the calculation, resonance parameters are re-evaluated. Statistical model parameters used for the calculation are also adjusted using available total cross section, capture
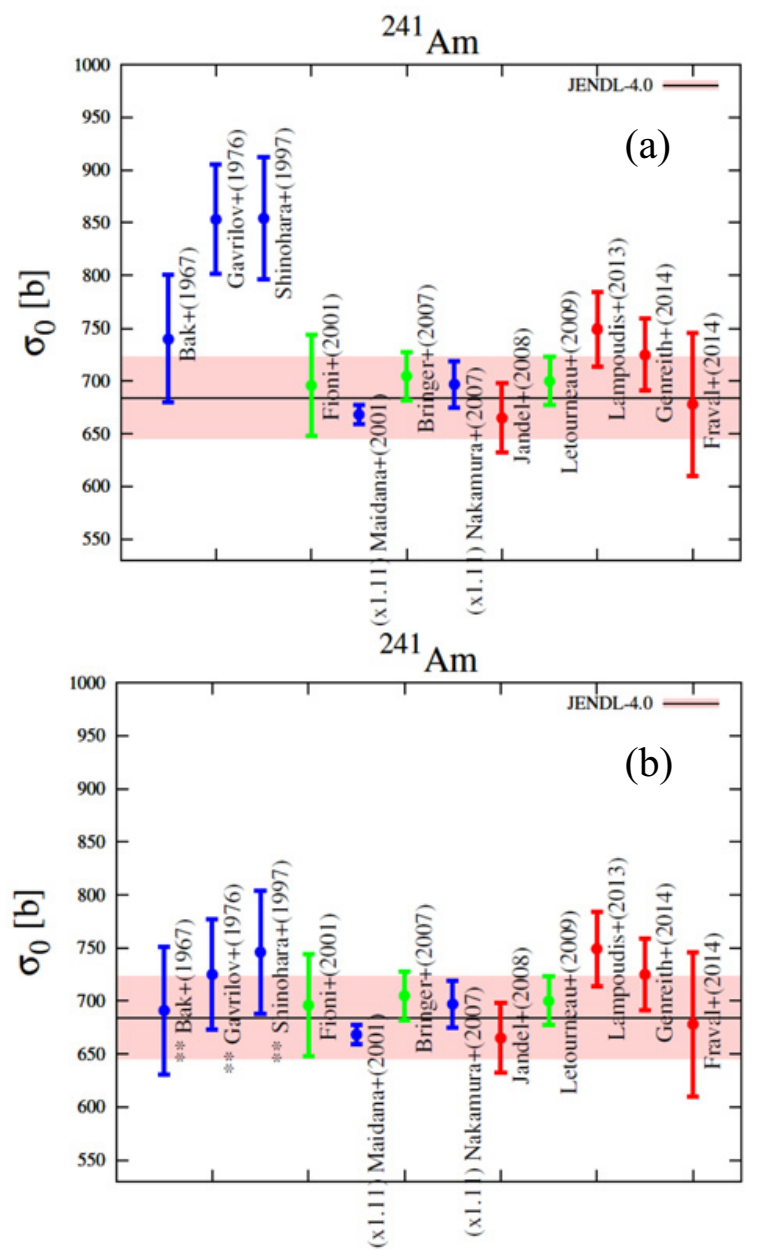

Figure 11. Capture cross section of ${ }^{241} \mathrm{Am}$ for thermal neutrons, before (a) and after (b) the systematic correction described in the text.

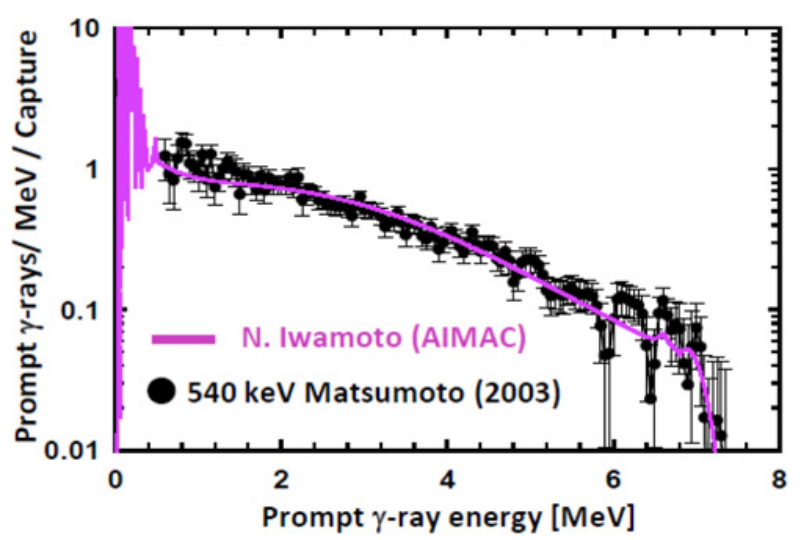

Figure 12. Prompt $\gamma$-ray spectrum for neutron capture reaction of ${ }^{99} \mathrm{Tc}$.

cross section, and prompt $\gamma$-ray yields. Figure 12 shows calculated and experimental prompt $\gamma$-ray yields [35] as an example of the evaluation. Details of the evaluation on capture cross sections and the developed methodology are described in [36].

\section{Conclusions}

Highlights of a Japanese nuclear data project (AIMAC) are outlined. Several independent measurement techniques 
are developed for improving measurement precision at JPARC/MLF/ANNRI and KURRI/LINAC facilities. Some of the newly developed techniques are shown to improve precision drastically. Effectiveness of combining the independent precise techniques is shown for identifying bias effects and improving accuracy. Importance of systematic re-evaluation with corrections of the identified bias effects is also demonstrated.

The present study includes the result of "Research and Development for accuracy improvement of neutron nuclear data on minor actinides" entrusted to the Japan Atomic Energy Agency by the Ministry of Education, Culture, Sports, Science and Technology of Japan (MEXT). HH deeply appreciates valuable discussions with P. Schillebeeckx.

\section{References}

[1] H. Harada, T. Baba, M. Igashira, H. Yamana, S. Raman, J. Nucl. Sci. Technol. Sup. 2, 366 (2002)

[2] M. Salvatores et al., WPEC-26, OECD/NEA (2008)

[3] H. Harada, et al., WPEC-31, OECD/NEA (2014)

[4] H. Iwamoto, K. Nishihara, T. Sugawara, K. Tsujimoto, J. Nucl. Sci. Technol. 50, 856 (2013)

[5] H. Iwamoto, K. Nishihara, R. Katano, K. Tsujimoto, JAEA-Research 2014-033 (2014)

[6] K. Shibata, O. Iwamoto, T. Nakagawa, et al., J. Nucl. Sci. Technol. 48, 1 (2011)

[7] H. Harada, O. Iwamoto, N. Iwamoto et al., EPJ Web of Conferences 93, 06001 (2015)

[8] A. Kimura, T. Fujii, S. Fukutani, et al., J. Nucl. Sci. Technol. 49, 708 (2012)

[9] K. Hirose, K. Furutaka, K.Y. Hara, et al., J. Nucl. Sci. Technol. 50, 188 (2013)

[10] H. Harada, M. Ohta, A. Kimura, et al., Nucl. Data Sheets 119, 61 (2014)

[11] E-I. Esch, R. Reifarth, E.M. Bond et al., Phys. Rev. C 77, 034309 (2008)

[12] M. Jandel, T.A. Bredeweg, E.M. Bond et al., Phys. Rev. C 78, 034609 (2008)

[13] K. Fraval, F. Gunsing, S. Altstadt et al., Phys. Rev. C 89, 044609 (2014)

[14] E. Mendoza, D. Cano-Ott, C. Guerrero et al., Phys. Rev. C 90, 034608 (2014)
[15] L. Lampoudis, S. Kopecky, O. Boulamd et al., Euro. Phys. J. Plus 128, 86 (2013)

[16] https://www.oecd-nea.org/science/wpec/ sg41/

[17] Y. Shibahara, et al., J. Radioanal. Nucl. Chem. 307, 2281 (2016)

[18] Y. Shibahara, et al., to be published in Proc. of ND2016 (S301)

[19] R.G. Helmer, J.C. Hardy, V.E. Iacob, et al., Nucl. Instru. Meth. A 511, 913 (2003)

[20] K. Terada, S. Nakamura, T. Nakao, et al., to be published in J. Nucl. Sci. Technol., doi: 10.1080/00223131.2016.1174167 (2016)

[21] K. Terada, et al., to be published in Proc. of ND2016 (R300)

[22] Y. Takahashi, H. Yashima, et al., to be published in Proc. of ND2016 (S033)

[23] A. Kimura et al., in preparation

[24] K. Mizuyama, et al., to be published in Proc. of ND2016 (S114)

[25] K. Kino, M. Furusaka, F. Hiraga, et al., Nucl. Instru. Meth. A 736, 66 (2014)

[26] T. Nakao, et al., to be published in Proc. of ND2016 (R130)

[27] T. Katabuchi, T. Matsuhashi, T. Terada, et al., Nucl. Instru. Meth. A 764, 369 (2014)

[28] T. Katabuchi, et al., to be published in Proc. of ND2016 (S111)

[29] T. Sano, et al., to be published in Proc. of ND2016 (S055)

[30] C.H. Westcott, W.H. Walker, T.K. Alexander, Proc. $2^{\text {nd }}$ Int. Conf. Peaceful Uses of Atomic Energy, Geneva 16, 70 (1958)

[31] H. Harada, S. Nakamura, T. Katoh, Y. Ogata, J. Nucl. Sci. Technol. 32, 395 (1995)

[32] S. Nakamura, M. Ohta, H. Harada, et al., J. Nucl. Sci. Technol. 43, 1500 (2007)

[33] G. Zerovnik, B. Becker, T. Belgya, et al., Nucl. Instru. Meth. A 799, 29 (2015)

[34] K. Mizuyama, N. Iwamoto, O. Iwamoto, published online in J. Nucl. Sci. Technol. (2016)

[35] T. Matsumoto, M. Igashira, T. Ohsaki, J. Nucl. Sci. Technol. 40, 61 (2003)

[36] N. Iwamoto, et al., to be published in Proc. of ND2016 (S185) 